\title{
latrogenic Complications During the Diagnostic Work-Up of an Inflammatory Cardiomyopathy
}

Hugo Antunes, ${ }^{\circledR}$ Júlio Gil, ${ }^{\circledR}$ Bruno Marmelo ${ }^{\circledR}$, Maria Luísa Gonçalves, ${ }^{\circledR}$ Maria Inês Pires, ${ }^{\circledR}$ João Miguel Santos, ${ }^{\circledR}$ Miguel Correia, José Costa Cabral ${ }^{\circledR}$

Centro Hospitalar Tondela-Viseu, Viseu - Portugal

\section{Abstract}

A 72-year-old woman was admitted for acute heart failure. The echocardiography revealed moderate depression of the left ventricular ejection fraction. Coronary disease was excluded by coronarography. Cardiac magnetic resonance showed predominantly left ventricular septal hypertrophy and severe depression of the left ventricular systolic function. There was also a bright, multifocal and patchy late gadolinium enhancement with subendocardial, mesocardial and subepicardial involvement, suggestive of sarcoidosis. Biochemical study, thoracic computed tomography and positron emission tomography were inconclusive for extra-cardiac sarcoidosis. Therefore, an endomyocardial biopsy was performed. The procedure was complicated by the development of complete atrioventricular block, requiring implantation of a cardiac resynchronization pacing device. A few days after device implantation, the patient developed fever. The echocardiography revealed extensive vegetations, and thus the diagnosis of a device-associated infective endocarditis was made. Even though antibiotic therapy was promptly started, the patient ended up dying. Biopsy results revealed lymphocytic myocarditis.

This case is paradigmatic because it shows how the etiologic diagnosis of dilated cardiomyopathy can be challenging. Non-invasive diagnostic exams may not

\section{Keywords}

Iatrogenic Disease / complications; Cardiomyopathies; Diabetes Mellitus; Hypertension; Endocardites; Cardiomyopathy, Dilated; Spectroscopy, Magnetic Resonance/methods. provide a definite diagnosis, requiring an endomyocardial biopsy. However, the benefits versus risks of such procedure must always be carefully weighted.

\section{Introduction}

The authors describe an intriguing case of dilated cardiomyopathy with left ventricular systolic dysfunction of recent onset. It is a paradigmatic case of how challenging the etiologic diagnosis of dilated cardiomyopathy can be. Sometimes, non-invasive imaging tests don't provide a definite answer. This paper also discusses the prognosis of cardiac device-related infective endocarditis and the importance of an early surgical intervention in gramnegative infections.

\section{Case Report}

A 72-year-old woman with a past medical history of diabetes mellitus and arterial hypertension was admitted for sudden-onset dyspnea. She had been complaining of fatigue and lower exercise tolerance over the previous four weeks. Physical examination showed hemodynamic stability, low peripheral oxygen saturation and signs of respiratory exhaustion. Lung auscultation revealed crackles in the lower two-thirds of both hemithoraces. The patient became clinically stable after non-invasive ventilatory support, along with diuretic and vasodilator therapy.

The electrocardiogram (ECG) showed sinus rhythm and complete left bundle branch block (LBBB). The transthoracic echocardiogram (TTE) revealed left ventricular dilation with a moderately depressed ejection fraction (left ventricular ejection fraction of $35 \%$ ) and preserved right ventricular systolic function.

Mailing Address: Hugo Antunes

Cardiology Department, Tondela-Viseu Hospital Centre - Av. Rei D. Duarte. Postal Code: 3504-509. Viseu - Portugal.

E-mail: hugo.ads.antunes@gmail.com 
There was no significant valve dysfunction or signs of pulmonary hypertension. Coronary disease was excluded by coronarography.

At discharge she presented with New York Heart Association (NYHA) class II heart failure. During outpatient follow-up, a cardiac magnetic resonance (CMR) was performed, confirming a moderate dilation of the left ventricle, asymmetric septal hypertrophy and severe depression of the systolic function, with akinesia of the mid and basal segments of the antero-septal wall. There were bright areas of late gadolinium enhancement (LGE) in multiple locations, affecting approximately $12 \%$ of the myocardial mass, including areas of nonhypertrophied myocardium (Figures $1 \mathrm{~A}-\mathrm{F}$ ). There was a predominantly subendocardial and mesocardial (though almost transmural) LGE area in the mid-segment of the anteroseptal wall; multiple confluent foci in the midsegment of the septal wall; subendocardial foci in the mid-segment of the inferior wall; and sub epicardial foci in the mid-segment of the infero-lateral wall.
The CMR findings were suggestive of non-ischemic cardiomyopathy, raising the suspicion of cardiac sarcoidosis. Corticosteroid therapy was initiated. Blood work revealed normal levels of alkaline phosphatase, angiotensin converting enzyme and inflammatory markers. Blood and urine calcium levels were also normal. A thoraco-abdomino-pelvic computed tomography did not reveal any lesions suggestive of sarcoidosis.

A ${ }^{18} \mathrm{~F}$-fluorodeoxyglucose $\left({ }^{18} \mathrm{~F}-\mathrm{FDG}\right)$ positron emission tomography (PET) revealed diffuse myocardial uptake, with focal areas of hypercaptation, with no unequivocal relationship with the LGE areas. The study was not conclusive because of the patient's inability to do a 12hour fasting due to a history of diabetes.

The patient presented with a dilated cardiomyopathy (DCM) with severe depression of left ventricular systolic function of recent onset and with no known cause, probably of an inflammatory nature. Considering a possible diagnosis of isolated cardiac sarcoidosis, which is a potentially treatable cause of DCM, an
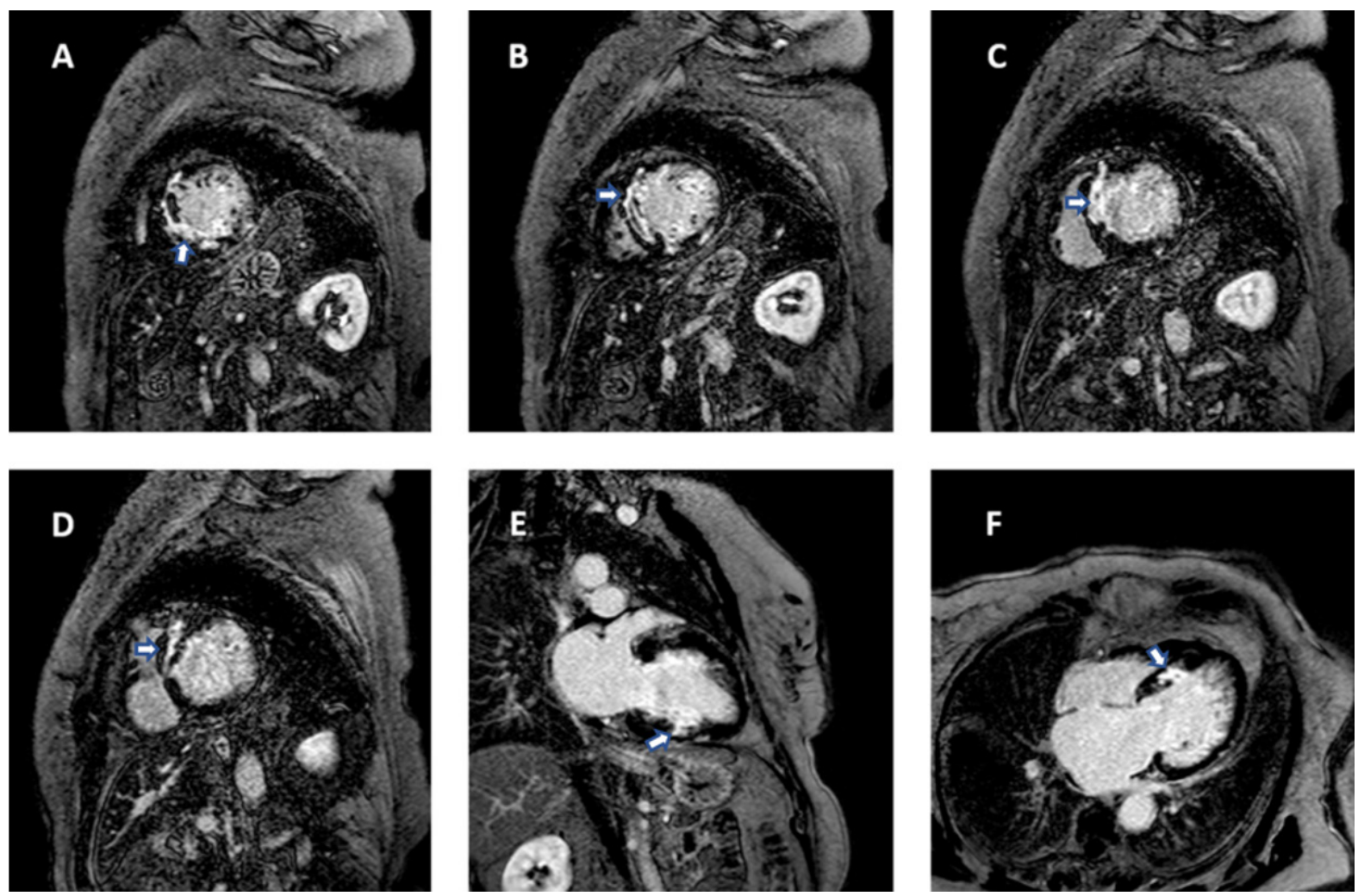

Figure 1 - Cardiac magnetic resonance revealing multifocal areas of late gadolinium enhancement (arrows), suggestive of nonischemic cardiomyopathy.

A-D: Short axis views, E: 2-chamber view and F: 4-chamber view. 
endomyocardial biopsy (EMB) was performed. Three myocardial fragments were successfully collected from the right ventricle.

The EMB procedure was complicated by a thirddegree atrioventricular block (AV block), requiring temporary pacing. Four days after the biopsy, the AV block did not disappear. Considering the presence of severe left ventricular systolic dysfunction and LBBB, a cardiac resynchronization therapy pacemaker (CRT-P) was implanted.

Four days after discharge, the patient was readmitted complaining of malaise and fever. Physical examination showed hemodynamic and electrical stability, without signs of localized infection or heart failure (HF). Blood cultures were collected and a course of empirical antibiotic therapy with ceftriaxone was initiated. At day 3 of hospitalization, vancomycin was added to the regimen, and ceftriaxone was substituted with gentamycin due to persistency of fever. Blood cultures revealed Escherichia coli (E. coli) and methicillinresistant Staphylococcus aureus (MRSA) growth. A transesophageal echocardiography (TEE) revealed significant masses, suggestive of vegetations, the biggest measuring $32 \mathrm{~mm}$, attached to the pacemaker lead, tricuspid and mitral valve (Figure 2), causing severe tricuspid regurgitation and confirming the diagnosis of device-related infective endocarditis (IE).

An extensive endocarditis, with large vegetations, associated with valvular dysfunction was found, in addition to isolation of Gram-negative bacteria. Therefore, cardiac surgery was indicated. While awaiting surgical correction, the patient developed clinical deterioration, with progressive multiorgan failure. She ended up dying at day 21 of hospitalization.

After the patient's death, histopathological results of the EMB revealed morphological damage of cardiac myocytes with cytoplasmic heterogeneity. There was evidence of inflammatory cell infiltrate, including
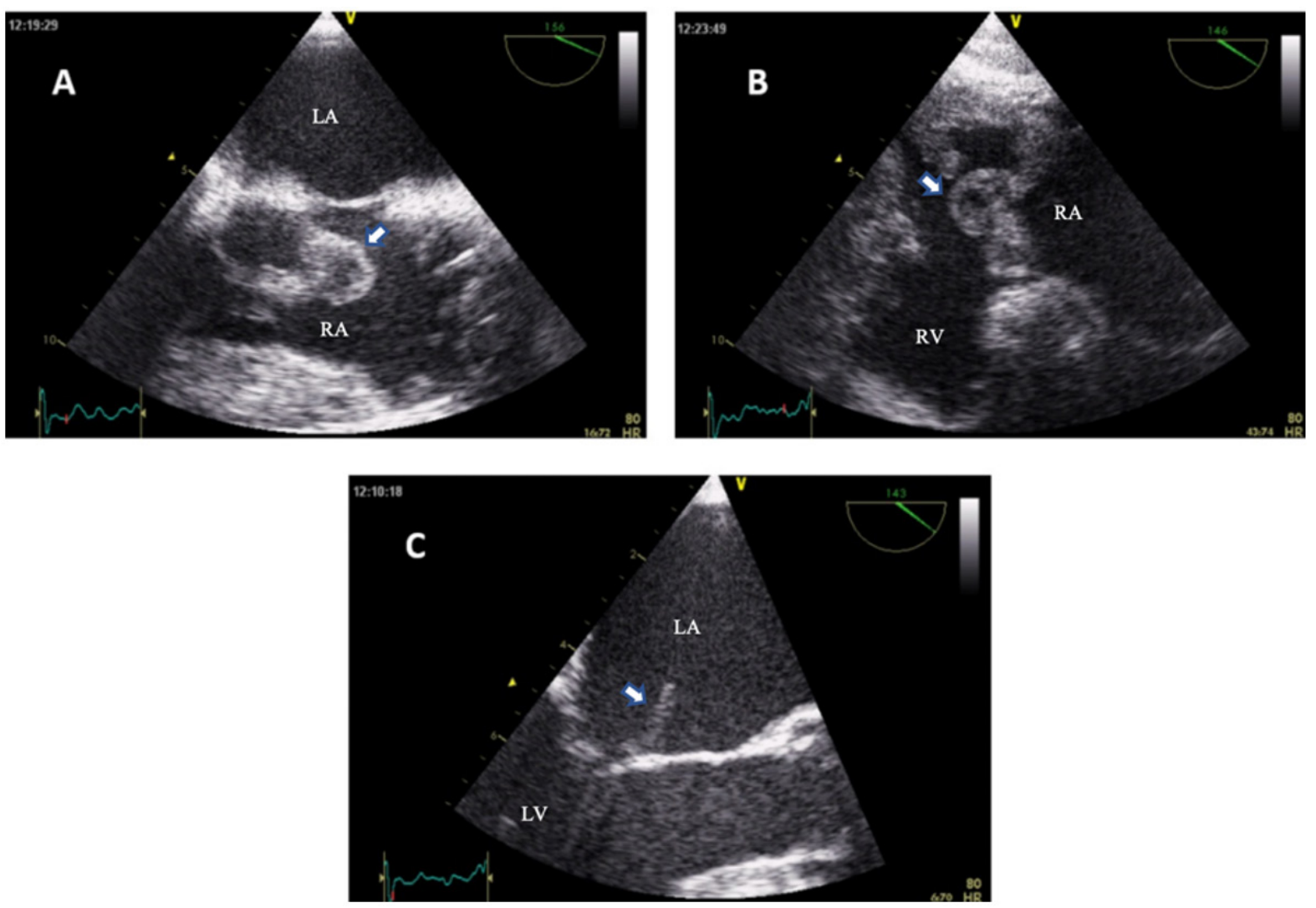

Figure 2 - Transesophageal echocardiography showing large vegetations (arrows).

$L A$ : left atrium; $R A$ : right atrium; $L V$ : left ventricle. 
lymphocytes and some neutrophils. PAS (periodic acid Schiff) and haematoxylin staining confirmed myocarditis of a probable infectious nature.

\section{Discussion}

The authors describe a case of a woman admitted for acute decompensation of HF. TTE showed left ventricular dilation and depression of systolic function. The patient was started on prognosis-modifying drugs, as tolerable. The CMR confirmed left ventricular dilation and systolic dysfunction, and significant areas of LGE in subendocardial, mesocardial and subepicardial regions. Corticosteroid therapy was initiated due to high suspicion of cardiac sarcoidosis, which was not confirmed by any of the test performed during diagnostic work-up. An EMB was then performed, but the procedure caused a series of events which culminated in patient's death. A complete AV block occurred, requiring temporary pacing and subsequent permanent pacing with CRT-P. Later, the patient developed fever, and had a diagnosis of IE.

This clinical case report demonstrates how noninvasive diagnostic exams may not provide a definite diagnosis. While cine-CMR showed segmental wall motion abnormalities, the main finding of MR was the LGE pattern. There was a multifocal and patchy pattern of LGE, with subendocardial, mesocardial and subepicardial involvement. There is no specific pattern of LGE on CMR for cardiac sarcoidosis. ${ }^{1-4}$ LGE is usually multifocal and patchy and is most commonly seen in mid-basal segments of the septum and lateral wall, with mid-myocardical and epicardial involvement, although it can be seen with transmural involvement also. ${ }^{1,2}$ The presence of LGE in cardiac sarcoidosis is known to be associated with a worse prognosis, with a higher risk of death, aborted sudden cardiac death and ventricular arrhythmia., ${ }^{3,5-7}$ Therefore, LGE pattern was compatible with cardiac sarcoidosis.

A ${ }^{18} \mathrm{~F}-\mathrm{FDG}$-PET was performed in attempt to confirm the diagnosis, since, among the non-invasive imaging methods, this technique has the highest sensitivity and negative predictive value for the diagnosis of cardiac sarcoidosis. ${ }^{3}$ The PET revealed diffuse myocardial uptake, with areas of hypercaptation, but with no confirmed association with the LGE areas. The diagnosis of cardiac sarcoidosis depends on the presence of a positive EMB or on a confirmed diagnosis of extra-cardiac sarcoidosis, which was excluded in this patient. ${ }^{8}$ EMB has a low sensitivity in cardiac sarcoidosis, with reported rates of $20-40 \%$. This is probably due to the patchy nature of the disease, and hence, the fragments may not include the noncaseating granulomas necessary for the diagnosis. ${ }^{2,8}$ A posthumous diagnosis of presumable lymphocytic myocarditis was made according to the EMB.

According to the European Society of Cardiology, EMB may be considered in the setting of unexplained, new-onset HF of 2 weeks to 3 months' duration, associated with a dilated left ventricle, without new ventricular arrhythmias or Mobitz type II second- or third-degree AV heart block, that responds to usual care within 1 to 2 weeks. ${ }^{9}$ The authors decided to proceed with EMB since we were in the presence of a DCM with recent onset $\mathrm{HF}$ and unknown cause, not responsive to usual treatment and with hemodynamic deterioration. In addition, the main diagnostic hypothesis was isolated cardiac sarcoidosis, which requires EMB for a definitive diagnosis. The pathological finding of lymphocytic myocarditis ranges from $0 \%$ to $63 \%$ in patients with newonset HF of 2 weeks to 3 months' duration, associated with a dilated left ventricle. ${ }^{9}$ The reported complication rate of EMB is $<1 \% .{ }^{9}$ Acute complications include pericardial tamponade, ventricular or supraventricular arrhythmias, heart block, pneumothorax, and damage to the tricuspid valve. ${ }^{9}$ Patients with LBBB have a higher risk of heart block. ${ }^{9}$

Unfortunately, the patient developed an extensive IE, probably worsened by the concurrent immunosuppressive therapy. Treatment of this condition requires prolonged antibiotic therapy and complete hardware removal and is associated with a high mortality rate. ${ }^{10}$ Transvenous lead extraction is usually the recommended approach since this procedure is associated with lower risks than surgery.${ }^{10}$ However, to this patient, cardiac surgery was indicated due to the following reasons: (1) presence of tricuspid dysfunction requiring repair; (2) large vegetations (> $20 \mathrm{~mm}$ ); (3) blood cultures indicating MRSA and E. coli; the latter is a gram-negative bacterium, to which the recommended therapy is early surgery. ${ }^{10}$ Unfortunately, the patient was not able to survive long enough, corroborating the high mortality associated with cardiac device-related IE.

This case is interesting since it is representative of the challenge it is to establish a definite diagnosis in the study of DCM. The investigation of the etiology is crucial when inflammatory disease is strongly suspected, and there is potential for recovery. It is also important not to overlook a rare diagnosis, such as isolated cardiac 
sarcoidosis. EMB may be the only procedure that can provide definite answers in some cases, but it is not devoid of serious complications. Similarly, cardiac device-related IE is a severe disease associated with high mortality which requires prolonged antimicrobial regimens and device removal. Patients with isolation of gram-negative specimens in blood cultures in the context of IE may benefit from early surgical intervention.

\section{Author contributions}

Conception and design of the research: Antunes $\mathrm{H}$. Analysis and interpretation of the data: Antunes $\mathrm{H}$. Writing of the manuscript: Antunes H. Critical revision of the manuscript for intellectual content: Antunes $\mathrm{H}$, Gil J, Marmelo B, Gonçalves ML, Pires MI, Santos JM, Correia M, Cabral JC.

\section{References}

1. Satoh H, Sano M, Suwa K, Saitoh T, Nobuhara M, Saotome M, et al. Distribution of late gadolinium enhancement in various types of cardiomyopathies: Significance in differential diagnosis, clinical features and prognosis. World J Cardiol 2014;6(7):585-601.

2. Birnie DH, Nery PB, Ha AC, Beanlands RS. Cardiac Sarcoidosis. J Am Coll Cardiol 2016;68(4):411-21.

3. Aggarwal NR, Snipelisky D, Young PM,Gersh BJ, Cooper LT, Chareonthaitawee P. Advances in imaging for diagnosis and management of cardiac sarcoidosis. Eur Heart J Cardiovasc Imaging. 2015;16(9):949-58.

4. Patel MR, Cawley PJ, Heitner JF, Klem I, Parker MA, Jaroudi WA, et al. Detection of myocardial damage in patients with sarcoidosis. Circulation 2009;120(20):1969-77.

5. Nadel J, Lancefield T, Voskoboimik A, Taylor AJ. Late gadolinium enhancement identified with cardiac magnetic resonance imaging in sarcoidosis patients is associated with long-term ventricular arrhythmia and sudden cardiac death. Eur Heart J Cardiovasc Imaging 2015;16(6):634-41.

6. Hulten E, Agarwal V, Cahill M, Cole G, Vita T, Parrish S, et al. Presence of Late Gadolinium Enhancement by Cardiac Magnetic Resonance Among Patients With Suspected Cardiac Sarcoidosis Is Associated With Adverse

\section{Potential Conflict of Interest}

No potential conflict of interest relevant to this article was reported.

\section{Sources of Funding}

There were no external funding sources for this study.

\section{Study Association}

This study is not associated with any thesis or dissertation work.

\section{Ethics approval and consent to participate}

This article does not contain any studies with human participants or animals performed by any of the authors.

Cardiovascular Prognosis: A Systematic Review and Meta-Analysis. Circ Cardiovasc Imaging 2016;9(9):e005001

7. Greulich S, Deluigi CC, Gloekler S, Wahl A, Zurn C, Kramer U, et al CMR imaging predicts death and other adverse events in suspected cardiac sarcoidosis. JACC Cardiovasc Imaging 2013;6(4):501-11.

8. Okada DR, Bravo PE, Vita T. Isolated cardiac sarcoidosis: A focused review of an under-recognized entity. J Nucl Cardiol.2018;25(4):1147.

9. Cooper LT, Baughman KL, Feldman AM, Frustaci A, Jessup M, Kuhl $\mathrm{U}$, et al. The role of endomyocardial biopsy in the management of cardiovascular disease: a scientific statement from the American Heart Association, the American College of Cardiology, and the European Society of Cardiology. Endorsed by the Heart Failure Society of America and the Heart Failure Association of the European Society of Cardiology. Eur Heart J 2007;28(24):3076-93.

10. Habib G, Lancellotti P, Antunes MJ, Bongiorni MG, Casalta JP, Del Zotti F, et al. 2015 ESC Guidelines for the management of infective endocarditis: The Task Force for the Management of Infective Endocarditis of the European Society of Cardiology (ESC). Endorsed by: European Association for Cardio-Thoracic Surgery (EACTS), the European Association of Nuclear Medicine (EANM). Eur Heart J 2015;36(44):3075-128. 